\title{
Using DNA Computing in Classification
}

\author{
Abd El-Menem Abd El- \\ Bary \\ Arab Academy for Science, \\ Technology and Maritime \\ Transport (AASTMT), \\ Alexandria, Egypt
}

\author{
Roshdy AbdelRassoul \\ SM, IEEE \\ Arab Academy for Science, \\ Technology and Maritime \\ Transport (AASTMT), \\ Alexandria, Egypt
}

\author{
Aya Mohamed El-Ebshihy \\ Arab Academy for Science, \\ Technology and Maritime \\ Transport (AASTMT), \\ Alexandria, Egypt
}

\begin{abstract}
This paper presents a DNA based classification technique. It exploits the properties of the DNA as a quaternary logic with the advantages of better storage, better accuracy, and shorter time compared to binary logic. Several examples are employed to demonstrate using the DNA as a new classification method for differentiation between closely related objects such as boys and girls also we used neural network to demonstrate better accuracy and predict some objects for example boys. In addition the explanation of DNA computing, Boolean Circuit using DNA, Molecular Beacons, also DNA logic gates and some applications using DNA computing. Several programming tools are used to illustrate that using the MATLAB program.
\end{abstract}

\section{General Terms}

DNA computing.

\section{Keywords}

DNA, DNA Computing, DNA Logic Gates, Molecular Beacons (MB).

\section{INTRODUCTION}

"Nano computers" devices that already exist in nature played an important role in all living things. But they're uncontrolled by humans. For example we won't be able that can program a tree to calculate the digits of pi [1]. So the idea of using DNA has been appeared that shows its ability in storing and processing information took off in 1994 when a California scientist firstly used DNA in a test tube for solving a simple math problem [1]. From this time a lot of research groups have proposed designs for DNA computers, and those attempts have relied on an energetic molecule called ATP for fuel. The DNA computer seems to be as a clear water solution in a test tube. There is no mechanical device. A trillion biomolecular devices could fit into a single drop of water. Instead of showing up on a computer screen, results are analyzed using a technique that allows scientists to see the length of the DNA output molecule. DNA computation uses DNA and molecular biology, Instead of traditional technologies using silicon-based computer. A single gram of DNA with volume of $1 \mathrm{~cm}^{3}$ can carry a lot of information as a trillion compact discs, approximately 750 terabytes now we'll explain the details of DNA \& DNA Computing in the next sections. [1].

\section{DNA and DNA COMPUTING}

DNA (deoxyribonucleic acid) is a dual-strand chaining composed of four nucleotides; the four nucleotides that form a strand of DNA are as follows: adenine(A), guanine $(\mathrm{G})$, cytosine $(\mathrm{C})$, and thymine $(\mathrm{T})$; they are known as bases too. The chemical structure of DNA (the famous double- helix) was discovered by James Watson and Francis Crick in 1953.
It consists of a specific ligament of two linear strings of bases. This ligament called complimentarily: adenine bonds with thymine (A-T) and vice versa (T-A), cytosine bonds with guanine $(\mathrm{C}-\mathrm{G})$ and vice versa (G-C). This is known as Watson-Crick complementary. Each DNA strand has two

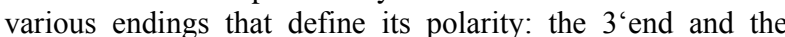
$5^{\prime}$ end. The double helix is an anti-parallel (two reversed of polarity) bonding of two integral strands [2]. The division of these nucleotides depends on the classification of the nitrogen bases which is:

- $\quad$ Purines (double-ringed structures): Adenine (A) and Guanine (G)

- $\quad$ Pyrimidines (single-ringed structures): Thymine (T) and Cytosine $(\mathrm{C})$

Each of which bonds together as a pair in the form of rungs in ladder [4]. Fig. (1) shows how is the bonding occur. [4]

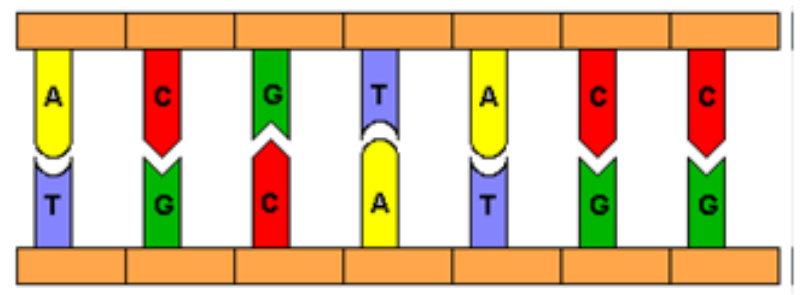

Fig. (1): Illustrates how is the bonding occur. [4]

The computational susceptibility of living systems has seized researchers for years. First of all, the emphasis has been on realization sides of living systems in computational devices. Examples are cellular automata, genetic algorithms, artificial neural networks, and artificial life. The argument has been that universal computational devices are able to simulate the attitude of physical, living systems through compatible programming. Thus, the tendency of invention has been from biology to computer science. Richard Feynman first introduced the molecular computation [1] in the early 1960s. This domain was firstly established by Leonard Adelman of the University of Southern California. In 1994, Adelman showed a proof-of-concept use of DNA as form of computation which was used to settle the seven-point Hamiltonian path problem. The electronic computers use two digits that are 0 and 1 called binary digits, while a DNA strand that composed of four-letter alphabet that is A, T, G and $\mathrm{C}$ which can hold much more information than earlier type of computers. Since the initial Adelman experiments, advances have been made, and various Turing machines have been proven to be constructible. Lipton proposed DNA experiments to solve the satisfiability problem. In 1997, Ouyen et al. approached a molecular biology instituted experiential solution to the "maximal clique" problem. In 
2000, Liu et al. designed a DNA model system, where a multi instituted encoding design is used in an approach to surface based DNA computation. In 2001, Wu analyzed and improved their surface-based method [1]. All their works use the tools of molecular biology, and all demonstrate the feasibility of carrying out computations at the molecular level. One of the formal frameworks for molecular computations is the Head's

splicing system, which gives a theoretical foundation for computing based on DNA recombination [1]. In the year 2004, Shapiro and co-workers constructed a DNA computer, coupled with an input and output module and is capable of diagnosing cancerous activity performance. The unique features were noted of DNA being an essential building block in the fields of super molecular chemistry, nanotechnology, Nano circuits, molecular switches, molecular devices and molecular computing [5].

\section{MOLECULAR BEACONS}

Molecular Beacons (MB) are single-stranded oligonucleotides hybridization plumb which hold a steam and ringed temple [8]. The loop owns complementary plumb series for the goaled one. This stem is formed by annealing with the complementary series found in either side of the plumb series a fluorophore and a quencher are connected to the two ends of the stem. The two octanes are kept in close proximity to each other by the stem, enabling fluorescence of the fluorophore tobe quenched through energy transfer and at this point the MB is "dark". When the MB encounters its goal DNA molecule, it submits a causal conformational reorganization that forces the forces the stem a part so that the fluorophore and quencher moves away. So there is a transformation from "dark" to fluorescence "bright" in MB. Molecular identification specificity is one of the main merits of MB. They are highly target-specific to the extent that they ignore goal series that vary even by a single nucleotide. Fig. (2) Illustrates the hybridization of the $\mathrm{MB}$ with the target. $\mathrm{MB}$ may be one the options of the inputs to the suggested Boolean circuit. [8]
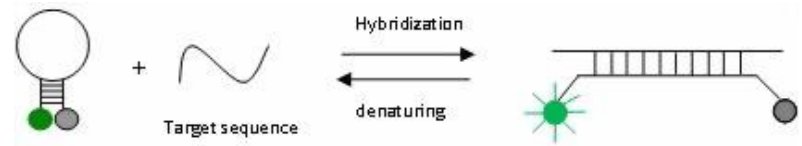

Fig. (2): hybridization of the MB with the target. [8]

\section{DNA SIMULATION OF BOOLEAN CIRCUITS}

Next we will describe how to simulate Boolean using bio molecular techniques. Boolean circuits merge the concept of wide ranged parallel signal processing and are repeatedly encountered in many parallel algorithms [6,7]. A DNA computation proceeds in two stages using encoding the problem by executing a solution space and applying molecular operations This simulation will occur using an the inputs and outputs as it will be discussed later complementary series found in either side of the plumb series a fluorophore and a quencher are connected to the two ends of the stem. The two octanes are kept in close proximity to each other by the stem, enabling fluorescence of the fluorophore to be quenched through energy transfer and at this point the MB is "dark". When the MB encounters its goal DNA molecule, it submits a causal conformational reorganization that forces the stem a part so that the fluorophore and quencher moves away. So there is a transformation from "dark" to fluorescence "bright" in MB. Molecular identification specificity is one of the main merits of MB. They are highly target-specific to the extent that they ignore goal series that vary even by a single nucleotide.

\section{DNA LOGIC GATES [3] \\ 4.1 NOT Gate}

The NOT gate, always known as an inverter, it is the simplest DNA-based logic gates that is because one input is supplied to the gate, and the output is the corresponding complementary sequence. As the output should evaluate "true" only in the presence of a "false" input, the base mixture provided to the gate contains the representative "true" sequence.

\subsection{XOR Gate}

The XOR gate is designed to give "true" if only one of the input sequences evaluates "true." With binary inputs, while XOR is defined as evaluating "true" if input values are opposite. In DNA-based logic gates, the XOR gate is the simplest design in the DNA Logic gates as there is no base chain needed to be provided to the gate.

\subsection{OR Gate}

The OR gate gives "true" if at least one of the gate inputs are "true". Introducing the "false" chain as DNA-Based enforcement of the XOR Gate. (a) One true and one false input results in a double- strand chain representing reality valuation. On the contrary, (b) two true inputs and (c) two false inputs do not create a double- strand chain. The base mixture will need at least one of the inputs be "true" to create a double- strand chain.

\subsection{NAND Gate}

The NAND gate gives "true" when inputs are not both "true," in the other meaning, if there is at least one input is false. The DNA-based NAND logic gate is the same as the OR gate that discussed previously, except the base sequence contains the sequence representing "true" rather than "false." Thus, at least one of the inputs must be "false" in order to create a double strand chain.

\section{DNA COMPUTING APPLICATIONS 5.1 Smart DNA Chips}

A gene term test with a single DNA chip can supply a visible display of how thousands of genes are 'expressed' jointly and a large amount of information on the genes. This field has a crucial modulation to vital pathogenetic applications such as drug design and disease classification. In order to improve the plenty, of new information made available by DNA chips, a

key challenge remains of how to design and develop intelligent machine learning techniques so as to effectively explore such a huge amount of information. The problem of over fitting is a leading concern with machine learning approaches to DNA chip data. These medical data are characterized by class imbalance, non-linear response, high noise, and large numbers of attributes. Intelligent DNA chips have been applied to the prediction and diagnosis of cancer, so that it expectedly helps to exactly predict and diagnose cancer. To precisely classify cancer we have to select genes related to cancer because extracted genes from DNA chips. have many noises [5]. This approach explored many features and classifiers using three benchmark datasets to systematically evaluate the performances of the feature selection methods and machine learning classifiers such as knearest neighbor, support vector machine. Also intelligent DNA chips were studied and it was shown that machine 
learning techniques offers available approach to identifying and classifying biologically relevant groups in genes and conditions [5]. The large width of DNA gene chip data does over fitting an ever present danger, particularly with powerful machine learning approaches.

\subsection{DNA Cryptography}

DNA Cryptography is a new cryptographic scope that uses DNA computing [9]. Some algorithms that are obtainable in DNA Cryptography own limitations in that they still use arithmetic module cryptography at some of their steps or they are biological laboratory trial based which is improper in the digital computing environment. In order to serve these security requirements, a lot of techniques and systems have been sophisticated in the mathematical cryptography for encoding and decoding the plain text. However, those mechanisms are overcome using DNA cryptography techniques. The DNA cryptography is arising domain in the DNA computing research .DNA cryptography plays an significant role in next generation security. Some algorithms that are obtainable in DNA Cryptography have restrictions in that they still use arithmetic modules for cryptography at some of their steps or they are biological laboratory experiment based which is improper in the digital computing environment. To overcome this problem secure, unique and dynamic DNA is used. The following steps will be required to conquer this problem: -

- DNA Encoding of complete character execution

- Dynamic Encoding Table Generation

- Unique sequence for encoding of every character of plaintext to DNA sequence

- Robustness of encoding

- Biological Process Simulation

- Dynamicity of encryption process

\subsection{DNA Computing algorithms for dual-} axis motion control and neural network A new way is suggested to treat with the dual-axis control of a multi-variables system with two induction motors [10]. Detection of solution the coupler problem of dual-axis platform is addressed by a neural net-based decoupling compensator and a sufficient condition ensuring closed- loop stability is derived. The information structure of DNA sequences can be extended to deal with parameter optimization and structure optimization problem in the current problem. The primary factors related to the tracking accuracy are fitness function and generation number of DNACA. The framework of implementation of DNACA was realized via a $\mathrm{NN}$ with the simplest structure. A meaningful fitness function and the reasonable generation number to tune the primary parameters. Fig. (3) shows the Coding architecture of DNACA-based neural networks [10].

\subsection{DNA Computing algorithms for dual- axis motion control and neural network}

A new way is suggested to treat with the dual-axis control of a multi-variables system with two induction motors [10]. Detection of solution the coupler problem of dual-axis platform is addressed by a neural net-based decoupling compensator and a sufficient condition ensuring closed- loop stability is derived. The information structure of DNA sequences can be extended to deal with parameter optimization and structure optimization problem in the current problem. The primary factors related to the tracking accuracy are fitness function and generation number of DNACA. The framework of implementation of DNACA was realized via a $\mathrm{NN}$ with the simplest structure. A meaningful fitness function and the reasonable generation number to tune the primary parameters. Fig. (3) shows the Coding architecture of DNACA-based neural networks [10].

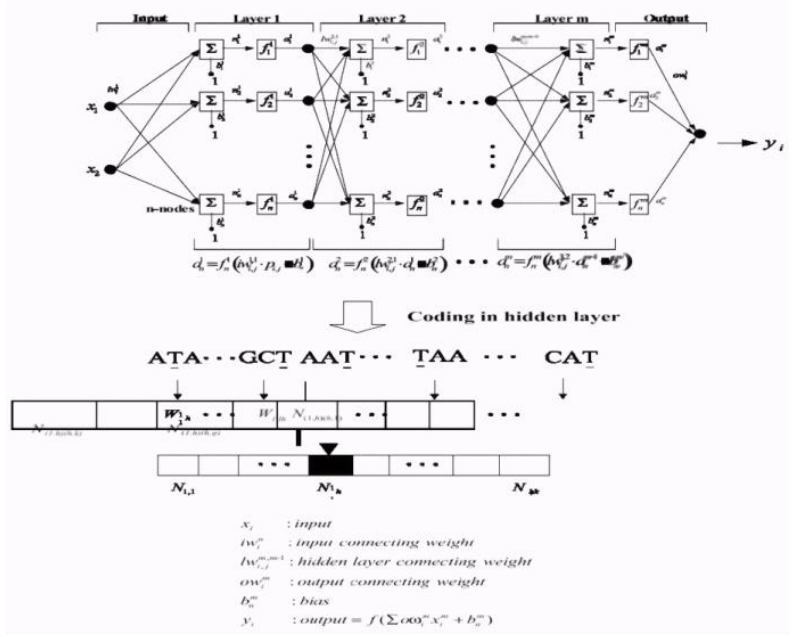

Fig. (3): Coding architecture of DNACA-based neural networks [10]

\subsection{DNA nano machines [1]}

DNA has been reconnoitered as a stellar tool for building large-scale nanostructures, constructing individual nano mechanical devices, and performing computations [11]. A variety of DNA nano mechanical devices have been previously constructed that demonstrate motions such as open/close, extension/contraction, and motors/rotation [12], interposed by extrinsic environmental variations such as the addition and removal of DNA fuel strands or the change of ionic composition of the solution [13]. The DNA walker could eventually be used to carry out computations and to strictly convey nanoparticles of material. The walker can be programmed in several paths in this direction. For example, information can be encoded in the walker segments as well as in the track so that, while performing motion, the walker jointly implements computation. In [14] an autonomous DNA walking device was designed in which a walker moves along a linear track unidirectional [15]. In [16] they constructed a DNA walking device controlled by DNA fuel strands. In [17] an independent DNA walking device and a separated DNA rolling device was designed that stir in an indiscriminate bidirectional fashion along DNA ways. Shin and Pierce [18] designed the DNA walker for molecular transport. Recently, Yin et al [14] encoded computational power into a DNA walking device embedded in a DNA lattice and therefore finished the design for an independent nano-mechanical device capable of universal computation and translational motion [19].

\subsection{A DNA Semantic Fusion Model for Remote Sensing Data [20]}

DNA has ability to store and process information. This information is determined by the sequence of four distinct bases (A, C, G, and T). An oligonucleotide is a short, singlestranded DNA molecule, and the complementary base pairing enables hybridization into a double stranded polymer. DNA computing, which is also called molecular computing, has major merits of in vivo computing and in vitro computing, 
such as huge parallelism, extraordinary information density and exceptional energy efficiency. In contrast to traditional silicon-based technology, DNA computing has the natural potency of semantic fusion and reasoning of huge data.

Nowadays, ontology has obtained more approval as one of semantic technologies to fix the problem of heterogeneous knowledge sharing.

DNA-based parallel computing takes a merit of many various DNA molecules to resolve the NP-complete problems in polynomial or even linear time, while exponentially growing time is needed in silicon-based computer. In this section, a DNA model is introduced for semantic fusion of the RSIs. It uses DNA computing and ontology technologies to enable the whole impersonation of the RSI's knowledge in linear time in any case the amount of data obtained.

Semantic network technology is used to fix information recognition problem.

In [21] a novel strategy to store digit information in DNA was proposed. In Church's work, all data blocks can be programmed into a bit stream and then encoded to thousands of oligonucleotides. But the successive diversion code (Perl) faces the challenge from big data.

$\mathrm{Xu}$ [22] provided a new DNA computing model for graph vertex coloring problem, which can effectively reduce the solution space by semi nested polymerase chain reaction. All these approximations described above reduction backing for semantic reasoning and quite concern has been given to big data, which have become the key problems of awareness sharing and semantic representation in the web environment.

In an attempt to overcome these difficulties, the researches propose here a novel DNA-based semantic fusion model as an expansion of the previous research for distributed data application in remote sensing field.

The researchers explain the effort which will be done in the paper as follows:

- Develop remote sensing data ontology with 1,264 concepts and 2,030 semantic relationships to annotate the RSIs.

- Encode arbitrary semantic properties, property values, semantic relationships and data types in DNA, and control the semantic information to steered directed acyclic graph.

- Evaluate the performance of the parallel transformation procedure versus the successive tactic with the Rest dataset

- Create an algorithm that takes merit of the biochemical reaction to fuse the semantic information.

\subsection{Hyper spectral remote sensing imagery [23]}

Hyper spectral remote sensing imagery provides richer spectral information than multispectral imagery and has been used to finely classify different ground materials. Compared with supervised classification, unsupervised classification for hyper spectral remote sensing imagery can discriminate different materials without prior information. However, due to the problem of the "curse of dimensionality "in highdimensional spaces, all of the dimensions may not be relevant to a given cluster. Therefore, hyper spectral data, with the larger volume, high dimensionality, and temporal and spatial spectral diversity, present a challenge for the traditional unsupervised clustering processing techniques such as kmeans, iterative self-organizing data (ISODATA), and fuzzy c-means clustering algorithms, which originate from multispectral imagery. In recent research, some artificial intelligence methods, such as the unsupervised artificial immune classifier, automatic fuzzy clustering using modified differential evolution (MoDEFC), and multi objective fuzzy clustering based on differential evolution and scheme with support vector machine classifier, have been developed to solve the multi-image clustering problem. These methods are used to estimate the class number and the distribution of clusters by multi cycle iterative optimization, but when these methods are introduced to process hyper spectral data, it is difficult to reach convergence and time-consuming to search the clusters in the sparse and skewed space. To avoid the problems resulting from the high dimensionality of hyper spectral imagery, band selection and dimension reduction are conducted before hyper spectral image processing. Many feature extraction and dimension reduction techniques have been developed in hyper spectral imaging, such as principal component analysis (PCA)

\subsection{Using DNA Computing in Classification and Prediction}

In the next section we will discuss how we use DNA computing in classification and prediction in order to get better accuracy and higher speed.

\subsection{Using DNA Computing in Classification and Prediction}

In the next section we will discuss how we use DNA computing in classification and prediction in order to get better accuracy and higher speed

\section{RESULTS AND DISCUSSION}

In this section we will use Dell Laptop Inspiron N4030, Intel ${ }^{\circledR}$ Core $^{\mathrm{TM}}$ i3 CPU M380@2.53GHz Processor, 2GHz RAM, 32 bit operating system, windows 7 ultimate copyright (C)2009 Microsoft Cooperation, MATLAB Version 7.7.0.471(R2008b)

\subsection{Classification between objects using MATLAB}

In this part we will use DNA to classify between objects for example boys and girls, sick and healthy people ... etc. and that occurs using logic gates which is AND, OR ,NAND,NOR that depends on ternary logic to give better accuracy and lower time. In the next section we'll determine the gender using DNA logic gates and compare it with Boolean logic gates so that we can get better accuracy and lower time

\subsubsection{Identifying Boys Only OR Girls Only}

Let us assume that we have four symbols named by $\mathrm{S} 1, \mathrm{~S} 2$, S3, S4 where S1 carries all the features that refers to boys gender while S4 refers to the gender is girls and the other two symbols carry one feature for both of the two genders so in this case we 'll use DNA symbols as follows:-

- S1equal ACGT while S4 is the complement of S1 so that $\mathrm{S} 4$ will be TGCA

- $\quad$ S2 and S3 refers to carrying one character for both genders 
- In our case we use S2 to carry one feature for boys while S3 for girls

- So that S2 will be equal to TCGA and S3 will ne AGCT

- $\quad$ Then we convert DNA bases into binary numbers so that we would be able to write our code

- Then we convert binary numbers into decimal one

- Therefore $\mathrm{A}=1\left(\begin{array}{ll}0 & 1\end{array}\right), \mathrm{C}=2\left(\begin{array}{ll}1 & 0), \mathrm{G}=1\left(\begin{array}{ll}0 & 1\end{array}\right), \mathrm{T}=3\left(\begin{array}{ll}1 & 1\end{array}\right)\end{array}\right.$

- $\quad$ so that we can use our threshold value to be equal to 0.5 to realize all values to be more than threshold

- $\quad$ which means upon to given bases value we 'll get $\mathrm{S} 1$ cause it'll be greater than threshold value

- $\quad \mathrm{S} 1=00110111$ that refers to ACGT so that we could determine boys gender by it.

Table 1. Comparison between DNA AND Gate and Boolean AND Gate

\begin{tabular}{|c|c|c|}
\hline Gates Type & Accuracy & Time \\
\hline DNA AND Gate & $84 \%$ & $0.07 \mathrm{Sec}$. \\
\hline $\begin{array}{c}\text { Boolean AND } \\
\text { Gate }\end{array}$ & $50 \%$ & $0.20 \mathrm{Sec}$. \\
\hline
\end{tabular}

- While applying Boolean Logic Circuits we can get $\mathrm{S} 1=0110$ that refers to $\mathrm{XY}$ so that we determine boys gender as discussed above with accuracy and time.

- In case of applying values lower than threshold we will get $\mathrm{S} 4=00000000$ that refers to TCGA determining girls gender

- Also apply Boolean logic circuit to get $\mathrm{S} 2=0000$ that refers to girls.

\subsubsection{Identifying Boys OR Girls}

- We can get S3 that refers to one features of boys if and only if the threshold value higher than or equal 0.5

- Also we can get S2 that refers one features of girls if and only if the threshold value lower than or equal 0.5

Table 2. Show main comparison between DNA OR Gate and Boolean OR Gate

\begin{tabular}{|c|c|c|}
\hline Gates Type & Accuracy & Time \\
\hline DNA OR Gate & $87 \%$ & $0.09 \mathrm{Sec}$. \\
\hline Boolean OR Gate & $55 \%$ & $0.30 \mathrm{Sec}$. \\
\hline
\end{tabular}

\subsubsection{Classification and Prediction using neural network}

In this case we will using feed forward neural networks to get more accuracy and achieve lower time to classify and predict the classified data to ensure that the network trained well .Our parameters will be as the following:-

- Learning rate: 0.06

- No. of epochs :1000

- Training rate $: 50$

- $\quad$ Goal :0.03
In the next examples we will use DNA computing compared to neural network according to several parameters which accuracy, time, no. of iterations, error in order to reach our goal.

Table 3. Show main comparison between DNA computing and neural network

\begin{tabular}{|c|c|c|c|c|c|}
\hline $\begin{array}{c}\text { Main } \\
\text { points }\end{array}$ & $\begin{array}{c}\text { Accur } \\
\text { acy }\end{array}$ & Time & $\begin{array}{c}\text { No. of } \\
\text { iteratio } \\
\text { ns }\end{array}$ & Error & $\begin{array}{c}\text { Learning } \\
\text { rate }\end{array}$ \\
\hline $\begin{array}{c}\text { DNA } \\
\text { Computi } \\
\text { ng }\end{array}$ & $99 \%$ & 0.02 & 421 & 0.002 & $\sim 0.06$ \\
\hline $\begin{array}{c}\text { Neural } \\
\text { Network }\end{array}$ & $97 \%$ & 0.07 & 990 & 0.033 & $\sim 0.16$ \\
\hline
\end{tabular}

Fig. (4) shows the result of applying Neural Network in normal case compared to DNA computing, while Fig. (5) shows that DNA computing gives better performance compared to neural network.

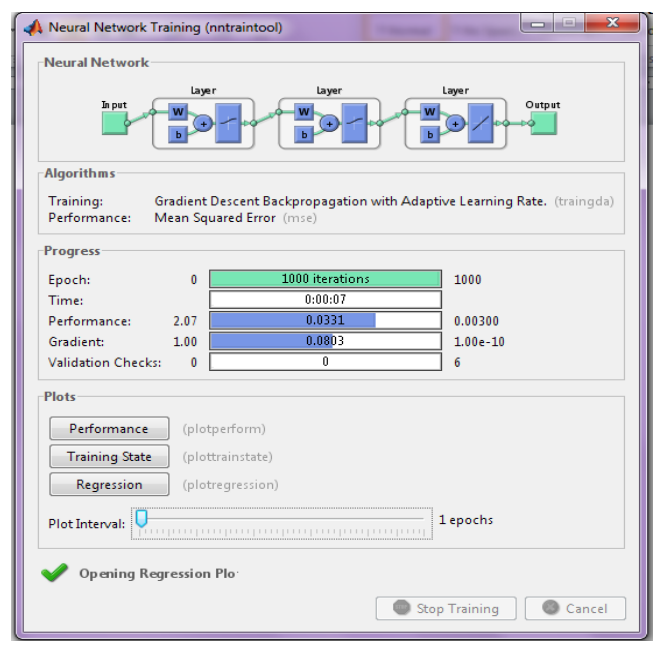

Fig 4.a

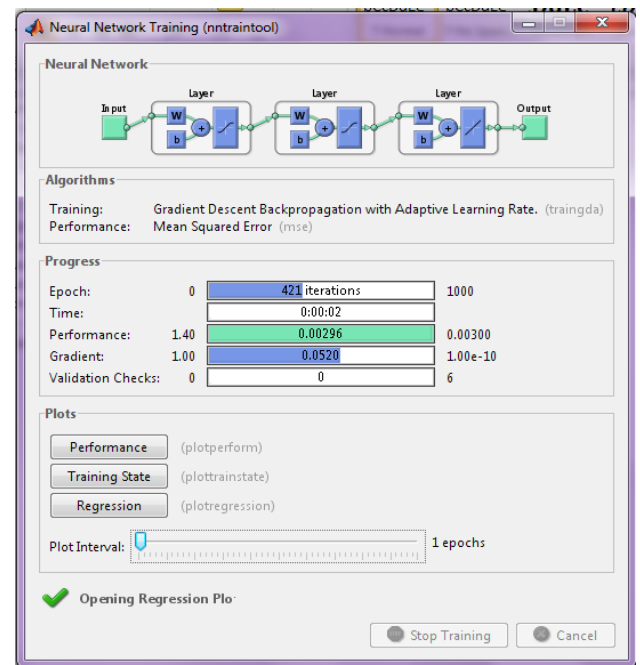

Fig 4.b

Fig. (4): Result of applying Neural Network in normal case compared to DNA computing 
Fig. (6) shows a comparison between the accuracy of achieving the target using DNA computing and neural network.

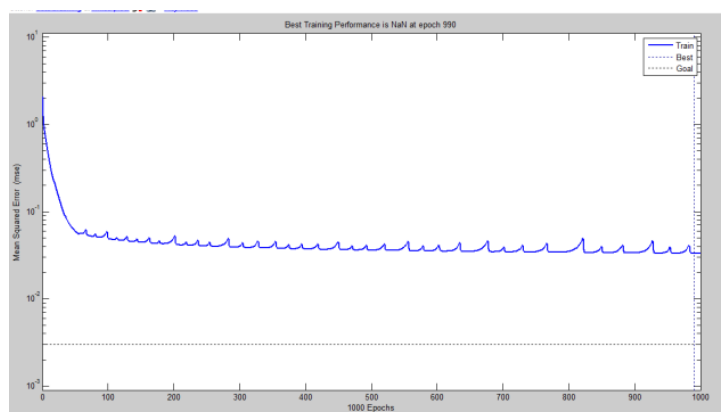

Fig 5.a

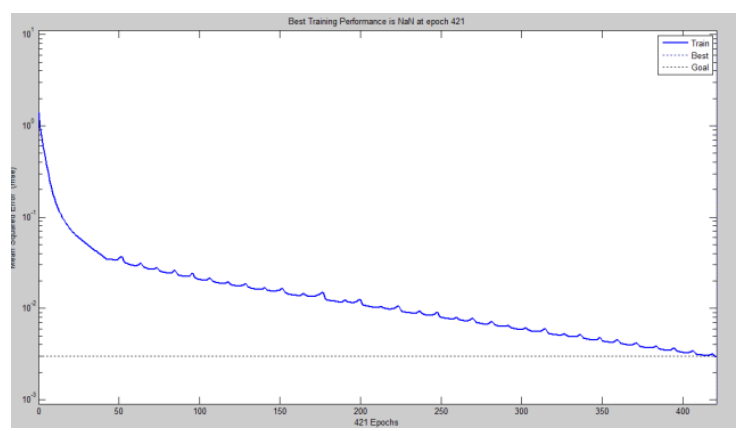

Fig 5.b

Fig. (5): performance of DNA computing compared to neural network

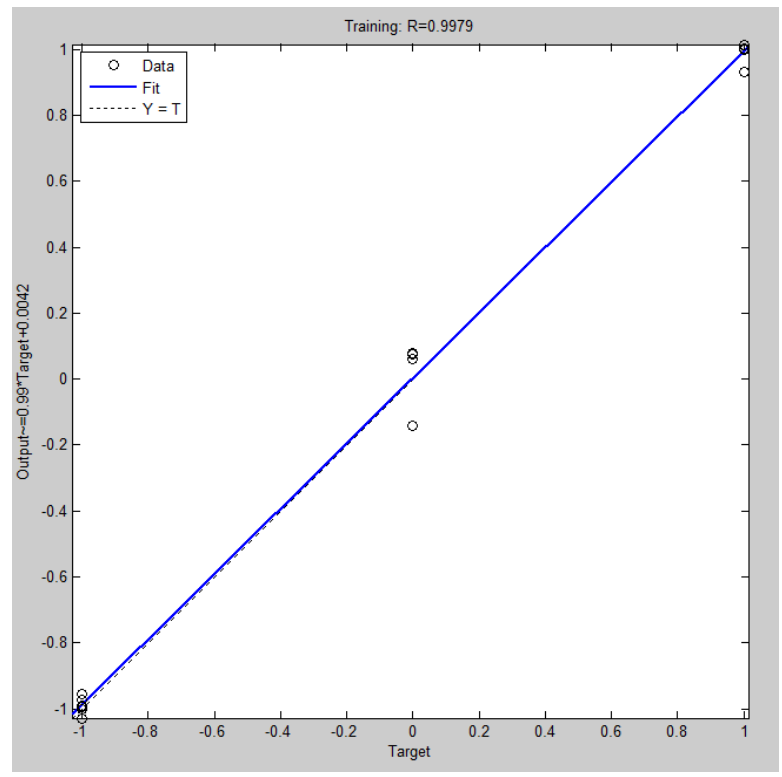

Fig 6.a

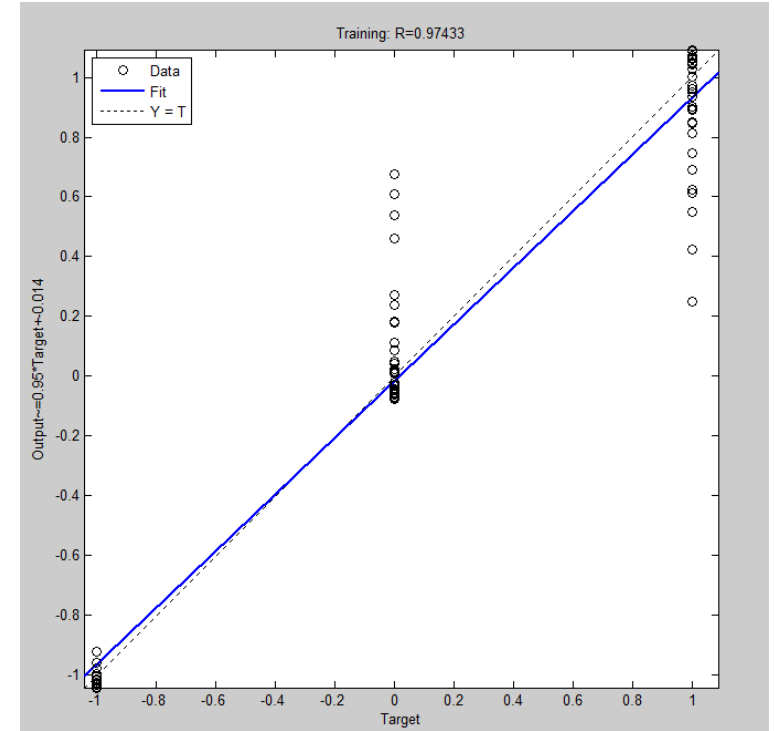

Fig 6.b

Fig. (6): Accuracy of achieving the target of DNA computing compared to neural network

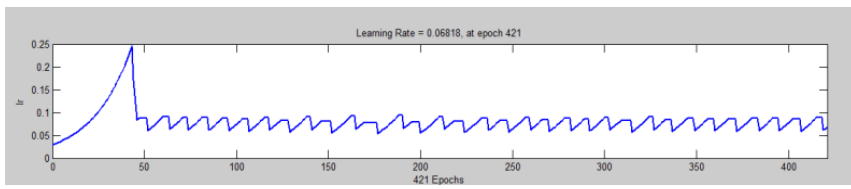

Fig 7.a

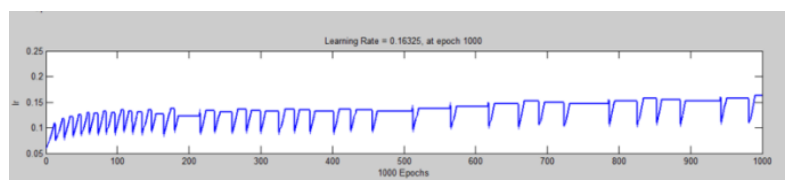

Fig 7.b

Fig. (7): Ability of DNA computing to learn the network compared to neural network

\section{CONCLUSION}

This paper demonstrates the DNA computing and some of its important applications in classification and prediction of data presented. Utilizing the DNA as a quaternary logic gives the advantages of better storage, better accuracy, and shorter time compared to binary logic. MATLAB programming is used for demonstration. Comparison between DNA computing and neural network shows that DNA neural network gives smaller error within best time and shows that DNA gives also higher accuracy.

\section{REFERENCES}

[1] DNA Computation: Applications and Perspectives, Somnath Tagore, Saurav Bhattacharya, Md Ataul Islam and Md Lutful Islam, Journal of Proteomics \& Bioinformatics, JPB/Vol.3 Issue 7,www.omicsonline.com

[2] Chapter 2, Introduction to DNA Computing, http://shodhganga.inflibnet.ac.in/bitstream/10603/6428/8 /08_chapter\%202.pdf.

[3] C. M. Gearheart, E. C. Rouchka, "DNA-Based Active Logic Design and Its Implications", Journal of Emerging Trends in Computing and Information Sciences, Vol. 3, No. 5, May 2012. 
[4] Seminar Report On DNA Computing, P. Sugathan S, Cochin University of Science and Technology, Cochin.

[5] DNA computing: applications and challenges, Nanotechnology 17 : R27-R39,

http://www.researchgate.net/publication/230987513

[6] DNA Simulation of Boolean Circuits, M. Amos, P. E. Dunne, Department of Computer Science, University of Liverpool, Report number CTAG-97009.

[7] Ahrabian, H., A. Nowzari-Dalini, "DNA Simulation of NAND Boolean Circuits", Department of Mathematics and Computer Science, Faculty of Science, University of Tehran, Tehran, Iran.

[8] M. Arock, B. S. E. Zoraida, R. Ponalagusamy, ,An Efficient Algorithm for Constructing DNA Boolean Circuit, 2010, International Journal of Computer Applications (0975 - 8887), Volume 1 - No. 22.

[9] A Novel DNA Computing based Encryption and Decryption Algorithm, Noorul Hussain Ubaidur Rahmana, C. Balamuruganb, R. Mariappanc, India, International Conference on Information and Communication Technologies (ICICT 2014), Procedia Computer Science 46 ( 2015 ) 463 - 475.

[10] Evolutionary neural networks and DNA computing algorithms for dual-axis motion control, Ching-Huei Huang, Chun-Liang Lin, Department of Electrical Engineering, National Chung Hsing University, Taichung 402, Taiwan, ROC, Volume 24, Issue 7 , October 2011, Pages 1263-1273.

[11] R. Pool, A boom in plans for DNA computation, Science $268,1995,498-499$

[12] Kubo, K., Morimoto M, Komuro T, Yata H, Tsunoda N, Kanehisa H, Fukunaga T., "Effect of plolymetric and weight training on muscle-tendon complex and jump performance". Med Sci Sports Exerc., 2007, Oct., 9(10), 1801-10.

[13] Chee, M., Yang, R., Hubbell, E., Berno A, Huang, X. C., Stern, D., Winkler, J., Lockhart, D. J., Morris, M. S., Fodor, S. P., "Accessing genetic information with highdensity DNA arrays,", Science. 1996, Oct., 25, 274(5287), 610-4.

[14] Yin, P., Hao Yan, Xiaoju G. Daniel, Andrew J. Turberfield, John H. Reif, A, "Unidirectional DNA
Walker Moving Autonomously Along a Linear Track", Angewandte Chemie Volume 43, Number 37, Sept. 20, 2004, pp 4906-4911.

[15] Liu, J., Ben-Shahar, T. R., Riemer, D., Treinin, M., Spann, P., Weber, K., Fire, A., \& Gruenbaum, Y., "Essential roles for Caenorhabditis elegans lamin gene in nuclear organization, cell cycle progression, and spatia organization of nuclear pore complexes", Mol Biol Cell, 11, 3937-47, 2000, doi:10.1091/mbc.11.11.3937.

[16] Sherman, W. B., N. C. Seeman, "A Precisely Controlled DNA Biped Walking Device”, Nano letters, 2004, 4 (7), 1203-1207.

[17] Yin P., Andrew J. Turberfield, John H. Reif, "Design of Autonomous Unidirectional DNA devices", Chapter, in 'DNA Computing', Volume 3384 of the series Lecture Notes in Computer Science, Claudio Ferretti Giancarlo Mauri, Claudio Zandron (Eds.), Milan, Italy, June 7-10, 2004, 410-425

[18] Shin, J. S., N. A. Pierce, "synthetic DNA walker for molecular transport", J Am Chem Soc. 2004, Sep 8, 126(35), 10834-5.

[19] Kaplan, P. D., Thaler, D. S., Libchaber, A., "Parallel overlap assembly of paths through a directed graph", Proceedings of the 3rd DIMACS Workshop on DNA Based Computers, 2001, pp. 127-141

[20] Sun, H., Jian Weng, Guang chuang Yu, Richard H Massawe, "A DNA-Based Semantic Fusion Model for Remote Sensing Data", Guangzhou, People's Republic of China, (2013), PLoS ONE 8(10): e77090. doi:10.1371/journal.pone.0077090.

[21] Church GM, Gao Y, Kosuri S (2012) Next-generation digital information storage in DNA. Science 337, 1628.

[22] Xu J, Qiang X, Zhang K, et al. A parallel type of DNA computing model for graph vertex coloring problem. Proceedings of the IEEE 5th International Conference on Bio-Inspired Computing: Theories and Applications (BIC-TA '10); September 2010, pp. 231-235.

[23] Jiao, H., Yanfei Zhong, and Liangpei Zhang, “An Unsupervised Spectral Matching Classifier Based on Artificial DNA Computing for Hyper spectral Remote Sensing Imagery", IEEE Transactions on Geoscience and Remote Sensing, V. 52, No. 8, Aug. 2014, 4524- 4538. 